EPJ Web of Conferences 59, 11002 (2013)

DOI: $10.1051 /$ epjconf/20135911002

(C) Owned by the authors, published by EDP Sciences, 2013

\title{
Overview of the LIFE fuel cycle
}

\author{
S. Reyes ${ }^{1, a}$, D. Babineau ${ }^{2}$, R. Davis ${ }^{2}$, C. Taylor ${ }^{3}$, T. Anklam¹, M. Dunne ${ }^{1}$, \\ D. Flowers ${ }^{1}$, C. Gentile ${ }^{4}$, J. Latkowski ${ }^{1}$, V. Maroni ${ }^{5}$, J. Martinez-Frias ${ }^{1}$, \\ R. Miles ${ }^{1}$ and S. Willms ${ }^{3}$
}

${ }^{1}$ Lawrence Livermore National Laboratory, Livermore, CA, USA

${ }^{2}$ Savannah River National Laboratory, Aiken, SC, USA

${ }^{3}$ Los Alamos National Laboratory, Los Alamos, NM, USA

${ }^{4}$ Princeton Plasma Physics Laboratory, Princeton, NJ, USA

${ }^{5}$ Argonne National Laboratory, Argonne, IL, USA

\begin{abstract}
The Laser Inertial Fusion Energy (LIFE) engine is a laser-driven inertial fusion energy system being developed with the goal to deliver fusion power in the next decade. A pre-conceptual design is being developed for the LIFE fuel cycle, with the purpose of maximizing the potential safety advantages of fusion energy. Some key features of the LIFE fuel cycle include a high tritium fuel burn-up fraction, a relatively high tritium breeding ratio, low tritium permeation from the coolant/breeder, and limited tritium inventories throughout the facility. The present paper offers an overview the pre-conceptual design of the LIFE fuel cycle, including a summary of the development plan for the delivery of the related tritium processing equipment.
\end{abstract}

\section{INTRODUCTION}

While many fusion plant designs require large quantities of tritium for start up and operations (with estimates of 8 to $60 \mathrm{~kg}$ per GW power plant), a range of design choices made for the LIFE fuel cycle act to reduce the in-process tritium inventory. The high fractional burn-up in an IFE capsule $(\sim 30 \%)$ [1] relaxes the tritium breeding requirements, while the use of only milligram quantities of fuel per shot and choice of a pure lithium coolant [2] substantially reduce the amount of material entrained in the facility to less than a kilogram for the entire site. Additionally, the high solubility of tritium in the lithium coolant is expected to mitigate the need for development of permeation barriers in the engine cooling systems, normally required to control routine releases below the allowable regulatory limits.

The LIFE fuel cycle encompasses the engine equipment that recovers the un-spent and the bred fuel from the engine systems, the tritium processing equipment that processes such fuel and conditions it for target manufacturing, and finally, the target manufacturing and transport/injection equipment. In the following sections we describe the pre-conceptual design of the main systems involved in each one of these areas. The design of LIFE tritium processing equipment is based on the concept of "line replaceable units" (LRUs), allowing for high availability of the tritium systems and reduced tritium inventories in the facility. Figure 1 below shows a simplified block diagram of the LIFE Fuel Cycle.

\footnotetext{
ae-mail: reyes20@llnl.gov
}

This is an Open Access article distributed under the terms of the Creative Commons Attribution License 2.0, which permits unrestricted use, distribution, and reproduction in any medium, provided the original work is properly cited. 


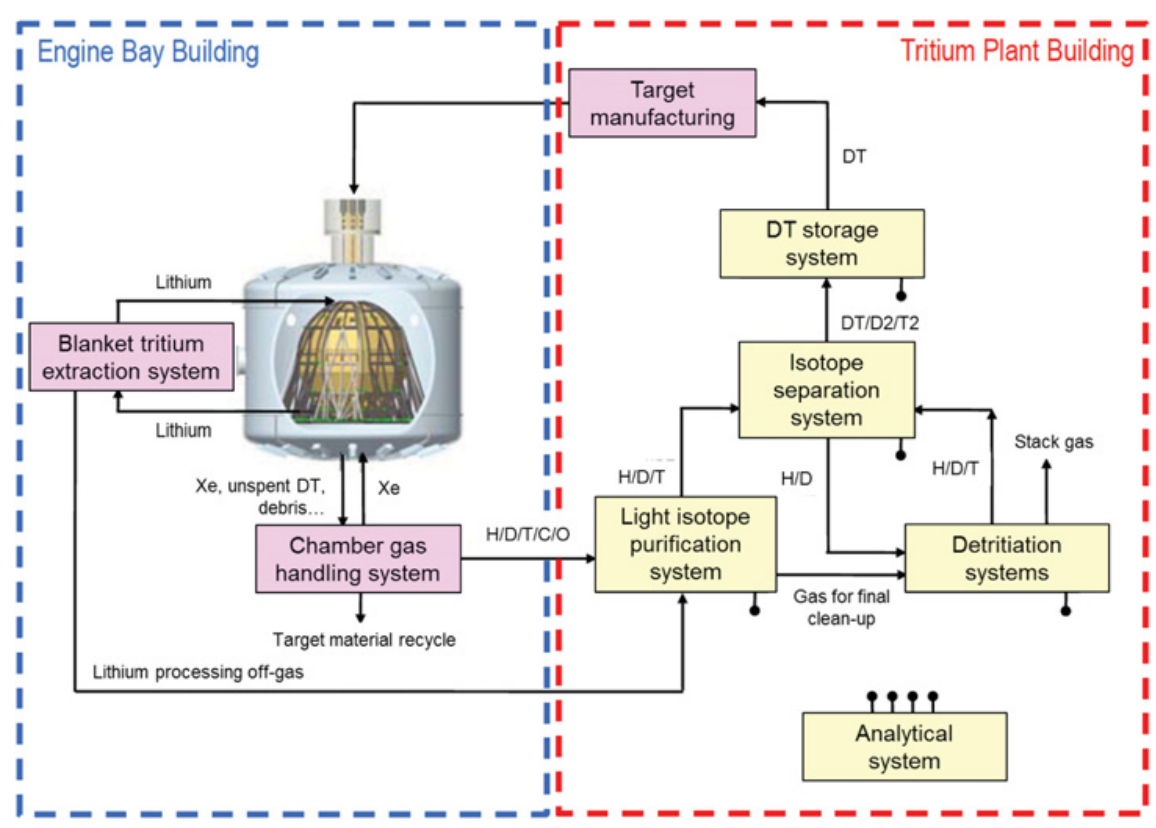

Figure 1. Simplified LIFE Fuel Cycle diagram.

\section{ENGINE TRITIUM SYSTEMS}

\subsection{Chamber gas handling system}

The fusion reaction inside the LIFE fusion chamber is created when 384 lasers focused onto an injected fuel target assembly: a capsule made of 50/50 mix of deuterium and tritium (DT) inside a hohlraum [3]. The LIFE chamber gas handling system (CGHS) treats the exhaust from the chamber and captures unburned DT from the fusion process. The chamber exhaust is primarily composed of the chamber fill gas (xenon), vaporized hohlraum material (lead), and trace amounts of carbon, hydrogen isotopes, and other target compounds. The first stage in the CGHS is gas cooling and lead separation by condensation. Once lead is removed, the chamber exhaust is cooled further and circulated back to the chamber using a blower or compressor. The recirculated gas is reheated to chamber operating conditions. A fraction $(\sim 10 \%)$ of the chamber exhaust is further processed for un-spent fuel recovery, which is then sent towards the processing systems in the tritium processing and target manufacturing area of the LIFE facility.

\subsection{Blanket tritium extraction system}

A molten salt extraction using centrifugal lithium/salt contactors and followed by electrolysis [2] is the proposed option for recovering the tritium bred in the lithium coolant/breeder, limiting the source term inventories in the cooling loops to just a few tens of grams.

This process was originally developed at Argonne National Laboratory and the different unit operations were tested at bench scale [4]. This technology is being adapted for LIFE tritium separation requirements, which include chemical compatibility of process equipment with lithium and tritium, and efficient separation of tritiated salt from the lithium to prevent cross contamination issues. The tritium extracted is sent directly towards the storage beds in the tritium processing systems, and from there supplied as required by the target manufacturing operations. 


\section{TRITIUM PROCESSING AND TARGET MANUFACTURING}

The LIFE tritium plant houses the tritium processing equipment that receives and processes the tritium from the two engine systems described in the previous section. It also provides space for the in-plant target manufacturing operations.

\subsection{Tritium processing}

\subsubsection{Light isotope purification system}

The system that receives the hydrogen isotope stream extracted from the chamber gas is the light isotope purification system (LIPS). Its most important functions are to break down hydrogen containing molecules, separate the hydrogen from non-hydrogen containing species, and to purify the hydrogen stream sufficiently for it to be sent to the isotope separation system. Additionally, this system will have to detritiate the process off-gases sufficiently for them to be sent to final clean-up by the detritiation systems before atmospheric release. At the pre-conceptual design stage, some technology candidates have been identified for the LIFE LIPS system, which include catalytic oxidation reactors, molecular sieve beds, permeators, and palladium membrane reactors (PMRs) [5], and most possibly will be a combination of these.

\subsubsection{Isotope separation system}

The main function of the isotope separation system (ISS) is to separate the hydrogen species into releasable and storable isotopic combinations. The ISS is expected to produce releasable gas streams $\left(\mathrm{H}_{2} / \mathrm{HD}\right)$ and storable $\mathrm{T}_{2}, \mathrm{D}_{2}$ and/or DT mixes with purities sufficient to be sent to the deuterium/tritium storage system. It is assumed that the releasable $\mathrm{H}_{2} / \mathrm{HD}$ streams will be sent to the detritiation system before atmospheric release through the elevated release point. The secondary function of the ISS is to recover and recycle $\mathrm{D}_{2}$ to be sent towards storage. The ISS is expected to be based upon one or more of the following technologies: thermal cycling absorption process (TCAP) [6], other chromatographic techniques [7], and/or cryogenic distillation [8]. Although TCAP currently appears as a promising option for LIFE, ongoing work is being devoted to refine the system requirements and identify possible interface issues.

\subsubsection{Deuterium/tritium storage system}

The DT storage system (DTS) receives and stores the DT, $\mathrm{D}_{2}$ and/or $\mathrm{T}_{2}$ as separated from the ISS, so as the $T_{2}$ extracted from the engine's lithium blanket, and supplies fuel as required by the target manufacturing systems. Additional functions of the DTS are to act as the major accountability center for the measurement of deuterium and tritium inventories in the LIFE facility, to receive and distribute all gasses to LIFE tritium wetted systems, to receive pure $T_{2}$ from the blanket tritium extraction system and to ship excess gasses ( $\mathrm{HD}, \mathrm{D}_{2}$ and $3 \mathrm{He}$ ) from the facility. Also, the DTS is expected to provide sufficient capacity to place the entire "at risk" tritium inventory onto beds to reach a "safe state" in case of accident/incidents in the tritium processing area. The LIFE DTS will utilize temperature controlled metal hydride bed technology for the storage of hydrogen isotopes [9].

\subsubsection{Analytical systems}

The analytical system interfaces with all the other systems within the LIFE tritium plant to provide inprocess tritium tracking and inventory management. It is expected that the tritium plant will switch to a non-processing mode when inventories need to be determined, and that tritium accountancy will be performed by the DTS in-bed calorimeters. Although the technologies for the tracking of radioactive gas 


\section{EPJ Web of Conferences}

species are well established [10], ongoing work is being devoted to the identification of LIFE-specific requirements.

\subsubsection{Detritiation systems}

The primary function of the detritiation systems (DS) is to ensure that all emissions from contaminated space and processes within LIFE are sufficiently decontaminated to allow for release to the environment through the elevated release point or stack. It is assumed that the DS will include the following subsystems: glove box detritiation, room detritiation, process gas detritiation, and final cleanup and stack. Ongoing work is being devoted to refine the identification of specific requirements for the LIFE DS. None the less, based on the available technologies that have been proven to date [11], it is expected that this system will be made up of a combination of catalytic oxidation reactors, molecular sieve beds, scrubber columns, and PMRs.

\subsection{Target manufacturing}

The central role of the in-plant target manufacturing activity is to deliver fuel targets in a physical form that complies with the requirements of implosion physics and in a sufficient quantity and price to supply affordable electricity to the grid. There are four major categories of target components/processes: capsule fabrication, hohlraum fabrication, membrane fabrication and the assembly operations. The current assumption is that capsule, membranes and hohlraum are manufactured separately and combined into subassemblies. Only the final assembly and DT fill is performed in the in-plant target manufacturing area.

\section{CONCLUSIONS}

The pre-conceptual design of the LIFE fuel cycle is presented here. Some key features include a high tritium fuel burn-up fraction $(\sim 30 \%)$, a relatively high tritium breeding ratio (possible through the use of a liquid lithium breeder), and low tritium permeation from the coolant/breeder (due to the high solubility of tritium in lithium). The use of only milligram quantities of fuel per shot and choice of a pure lithium coolant substantially reduce the amount of material entrained in the facility to less than a kilogram for the entire site. Ongoing work is being devoted to identify technology choices and potential interface issues. As the project evolves, all aspects related to tritium confinement and accountancy will remain as key considerations in the design of the LIFE tritium systems.

This work was performed under the auspices of the U.S. Department of Energy by Lawrence Livermore National Laboratory under Contract DE-AC52-07NA27344.

\section{References}

[1] M. Dunne, et al., Fusion Science and Technology, 60, 19 (2011)

[2] J. Latkowski, et al., Fusion Science and Technology, 60, 54 (2011)

[3] R. Miles, et al., Fusion Science and Technology, 60, 61 (2011)

[4] V.A. Maroni, et al., Nucl. Technol., 25, 83 (1975) and US Patent 3,957,597 (1976)

[5] S.A. Birdsell, R.S. Willms, Proceedings of the Symposium on Fusion Technology, 953 (1998)

[6] L.K. Heung, H.T. Sessions, X. Xiao, H.L. Mentzer, Fus. Sci. and Technol., 56, 1471 (2009) 


\section{IFSA 2011}

[7] C.H. Cheh, Journal of Chromatography A, 658-2, 283 (1994)

[8] J.R. Bartlit, R.H. Sherman, R.A. Stutz, W.H. Denton, Cryogenics, 19-5, 275 (1979)

[9] L.K. Heung, WSRC Report, WSRC-MS-2001-00179 (2001)

[10] D.R. Voorhees, R.L. Rossmassler, G. Zimmer, PPPL Report PPPL-CFP-3205 (1995)

[11] S. Beloglazov, et al., to appear in the Proceedings Tritium 2010 